\section{La Asociación Internacional de Productores de Tabaco ataca a la OMS}

\author{
Jaime Pérez-Martín ${ }^{1}$ \\ y Armando Peruga ${ }^{1}$
}

Palabras clave: tabaco, hábito de fumar.

\footnotetext{
1 Organización Panamericana de la Salud, Washington, D.C., EE.UU. Toda correspondencia debe dirigirse a Jaime Pérez-Martín al la siguiente dirección postal: 525 Twenty-third St., NW, Washington, DC 20037, USA.
}

Durante la última semana de febrero de 2002, la ciudad de Santiago de los Caballeros en la República Dominicana fue sitio de reunión de los cultivadores de tabaco de la Región de las Américas, agrupados bajo el nombre de Asociación Internacional de Productores de Tabaco (AIPT). El Gobierno de la República Dominicana estuvo representado por Manuel Álvarez, subdirector del Instituto de Tabaco de dicho país. Según los informes aparecidos en la prensa (1), la AIPT culpa a la Organización Mundial de la Salud (OMS) de haber reducido la superficie de tierra cultivada con la planta del tabaco en los países americanos y le atribuye "el intento de suspensión definitiva de la cosecha de la hoja" [sic], acusándola de no tomar en cuenta las consecuencias socioeconómicas de una reducción del consumo de tabaco. De acuerdo a estos mismos informes periodísticos, el secretario de la AIPT, el portugués Antonio Abrunhosa, destacó el papel del Gobierno de la República Dominicana en la defensa de los intereses de los cultivadores de la hoja en la negociación del Convenio Marco para el Control del Tabaco.

\section{¿Qué es la Asociación Internacional de Productores de Tabaco?}

La Asociación Internacional de Productores de Tabaco se describe a sí misma como "el organismo representativo de los cultivadores de tabaco de todo el mundo" y afirma hablar "en representación de los 33 millones de personas que participan en el cultivo y primera fase de procesamiento de la hoja del tabaco". No obstante, los documentos internos de la industria del tabaco que se hicieron públicos en los litigios recientes contra ella en Estados Unidos demuestran que la verdad dista de ser como la pinta la AIPT. No solo no se trata de una "asociación de comercio internacional independiente", sino que la AIPT es, en realidad, un vehículo de relaciones públicas creado por la industria tabaquera en los años ochenta para contrarrestar las iniciativas internacionales de control del tabaco y darle a la industria una cara humana y una voz dentro de los países en desarrollo $(2,3)$. Los documentos accesibles al público muestran que la AIPT ha recibido financiamiento de la industria tabaquera y está dirigida actualmente por una compañía de relaciones públicas con sede en el Reino Unido entre cuyos clientes figura la British American Tobacco (BAT) (4). 


\section{Los hechos y los argumentos}

1. Según la AIPT, la superficie sembrada con tabaco en México, Guatemala y América Latina en general ha descendido bruscamente, a tal punto que supuestamente ha habido una emigración hacia Estados Unidos de trabajadores ligados al cultivo del tabaco.

Los datos de la Organización de las Naciones Unidas para la Agricultura y la Alimentación (FAO) contradicen estas declaraciones de la AIPT (5). En 1990, la superficie sembrada en México con hoja de tabaco era de 21722 hectáreas $^{2}$ (ha), mientras que en 2000 dicha superficie era de 22674 ha, con un aumento de la producción en ese período de 33941 a 45164 toneladas métricas $(t)^{3}$. En Guatemala la producción fue de alrededor de $11100 t$ tanto en 1990 como en 2000.

Este ligero ascenso de la superficie sembrada de tabaco también se ha observado en todo el conjunto de América Latina en los últimos 10 años, período que corresponde al de mayor beligerancia por parte de la OMS en lo referente al tema del tabaco. La figura 1 revela esta tendencia ascendente de la superficie cultivada con oscilaciones cíclicas, es decir, ondas de crecimiento y reducción de dicha superficie, que se encuentra en la fase descendente de la oscilación desde 1998.

Los indicadores de la producción de hoja de tabaco en América Latina son de mayor relevancia económica que la superficie de tierra sembrada. Aunque esta última disminuyese, la producción de la hoja podría mantenerse o incrementarse aumentando el rendimiento por hectárea. De hecho, la producción en América Latina (figura 1) ha mostrado una tendencia ascendente en los últimos 12 años, pasando de $703421 t$ en 1990 a $868302 t$ en 2001. Esta producción se ha mantenido por encima de las $800000 t$ anuales desde 1997 y se encuentra entre las más altas de la historia. El rendimiento, representado por la producción por área de superficie cultivada, presenta en este período de 1990-2000 una tenden-cia general ascendente, aunque con oscilaciones.

2. La AIPT acusa a la OMS de querer acabar con la producción del tabaco.

A pesar de que las pruebas indican que la superficie de tierra sembrada con hoja de tabaco y la producción de dicha hoja han aumentado, la AIPT atribuye un falso descenso de estos indicadores a la política de la OMS. Ante esto hay que señalar que la OMS no quiere acabar con la producción del tabaco,

\footnotetext{
2 Una hectárea es un kilómetro cuadrado.

3 Una tonelada métrica equivale a $1000 \mathrm{~kg}$.
}

sino reducir notablemente el consumo de sus derivados. La OMS no tiene la intención de menoscabar los intereses económicos de los productores, sino defender la salud de la población, incluida la de los propios cultivadores de tabaco. Aunque la política de control del tabaco redujese el consumo de este al ritmo observado en los países que más éxito han tenido en sus campañas contra el tabaquismo, esta política todavía distaría de tener un impacto negativo sobre la situación económica de los agricultores.

Un estudio inédito reciente de la FAO indica que una reducción sostenida de un punto porcentual al año (2) de la prevalencia de tabaquismo durante los próximos 20 años no disminuirá las necesidades de producción de la hoja del tabaco. De acuerdo a este estudio, para el año 2010 se consumirán 7,1 millones de toneladas métricas de tabaco, en vez de los 6,5 millones consumidos a finales de los años noventa. En tal caso, aunque se reduzca la prevalencia del tabaquismo, el número absoluto de fumadores pasará de un total de 1300 millones en 2000 a 1400 millones en 2020. Por tanto, el consumo per cápita de productos del tabaco, que actualmente está disminuyendo en los países más desarrollados, continuará creciendo en los países en desarrollo.

Aun cuando fuese más rápida la reducción del tabaquismo, los efectos sobre la producción del tabaco se producirán a muy largo plazo y los gobiernos y cultivadores tendrán mucho tiempo para establecer planes encaminados a lograr una transición satisfactoria para todas las partes. Según el informe del Banco Mundial titulado La epidemia del tabaquismo (6), la reducción del consumo de tabaco que puedan ocasionar el Convenio Marco para el Control del Tabaco y las actividades antitabáquicas de la OMS y de sus Estados Miembros no afectará a esta generación de cultivadores.

El mismo informe del Banco Mundial indica que ni siquiera una brusca disminución del consumo de productos de tabaco tendría repercusiones macroeconómicas adversas en ninguno de los países de la Región de las Américas, ya que el dinero que moviliza el mercado del tabaco se desplazaría a sectores más productivos para la sociedad, pudiendo ello suponer una ganancia neta de fuentes de empleo en algunos países. Las conclusiones de estos informes independientes no se compaginan en absoluto con la catástrofe pronosticada por la AIPT.

3. Según estimaciones de la propia $\operatorname{AIPT}(7)$, el consumo anual de tabaco en el mundo es de 6,1 millones de toneladas métricas, cantidad muy inferior a la que producen los cultivadores.

La demanda mundial de hoja de tabaco para la manufactura de sus productos ha sido ampliamente superada por la producción en los últimos 10 años, 
FIGURA 1. Hectáreas (ha) de tierra cultivadas con hoja de tabaco, toneladas métricas (t) de hoja de tabaco producidas y toneladas métricas rendidas por hectárea cultivada ( $t /$ ha) en América Latina, 1990-2001

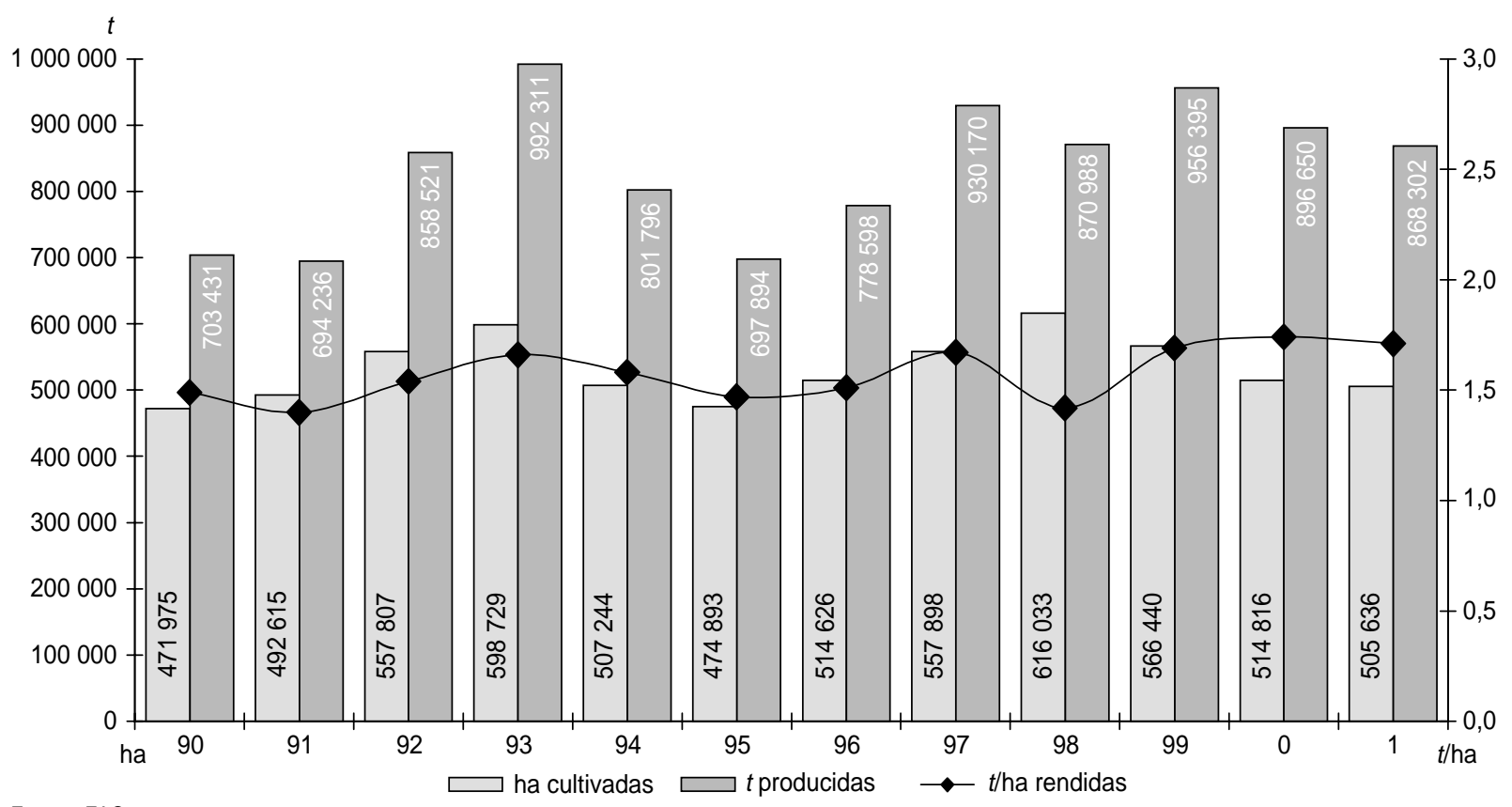

Fuente: FAO

que alcanzó un máximo de 9 millones de $t$ en 1997. La producción en el año 2000 fue de 6,8 millones de $t$, cifra que superó ampliamente las necesidades de consumo. Tales excedentes en manos de la industria hacen que los mismos cultivadores sean propensos a reducir la superficie sembrada para disminuir la producción y así conseguir que los precios suban o se mantengan estables. La propia AIPT atribuye a este motivo el supuesto descenso de la superficie sembrada y de la producción en los últimos años, según consta en su página web (8), donde describe los problemas de precio e inventario que suscitaron la disminución de la cosecha en el año 2000 en Brasil y Argentina. Como puede observarse, las declaraciones de la AIPT a la prensa anteriormente citadas (1) sobre la responsabilidad de la OMS en la disminución del cultivo de tabaco son muy diferentes de las que constan en su página web.

\section{¿Qué intereses defiende la AIPT?}

El consumo de tabaco causa casi 5 millones de muertes anuales en el mundo (9), de las cuales más de medio millón se producen en América Latina y el Caribe (10). De mantenerse la tendencia actual, en 2020 morirán en el mundo más de 8 millones de personas y más de $70 \%$ de ellas morirán en países en desarrollo (11). Además, 500 millones de las personas que viven actualmente morirán de enfermedades relacionadas con el tabaco (12). La OMS solamente busca evitar el dolor y sufrimiento que estas muertes puedan provocar. Por tanto, cabe preguntarse, ¿por qué tergiversa la AIPT las cifras que corresponden a la superficie de la tierra sembrada con hoja de tabaco, procurando que parezcan descensos catastróficos las oscilaciones cíclicas de una tendencia que en ningún caso es descendente? Si la AITP reconoce en sus documentos que las fluctuaciones de la producción de hoja de tabaco y de la superficie de la tierra cultivada con ella se deben a factores del mercado y al manejo del inventario acumulado por la propia industria tabacalera, ¿por qué busca que los cultivadores ataquen a la OMS y se opongan a sus políticas, que solo están encaminadas a defender el derecho de los pueblos a velar por su propia salud?

\section{SYNOPSIS}

\section{The International Association of Tobacco Growers attacks WHO}

The International Association of Tobacco Growers (IATG) has publicly blamed WHO's tobacco control policies for a decline in the amount of land where tobacco is grown in the Americas and for the fact that many farmers have lost a way to make a living. Contrary to 
these allegations, the surface of land where tobacco is grown and the production of tobacco have increased between 1990 and 2000 (from 471975 to 505636 hectares and from 703431 to 868302 metric tons, respectively). Furthermore, the IATG's internal documents point to the cause of the declining harvests observed in some countries as being the drop in prices that has been triggered by surplus production on a global scale.

According to internal documents belonging to the tobacco industry, the IATG is a public relations agency that was created by the industry as its spokesman in developing countries in an effort to curb tobacco control initiatives. WHO aims to reduce the use of tobacco products and the morbidity and mortality that are attributable to such use, a measure which will have no impact on the current generation of tobacco growers because, even if the prevalence of smoking does decline, the total number of smokers will continue to grow due to the overall increase in the size of the population.

\section{REFERENCIAS}

1. Jiménez P. Productores critican campaña negativa de OMS contra tabaco. Listín Diario.com.do. [Publicación seriada en línea]. Sabado 23 de Febrero de 2002 [citado el 4 de octubre de 2002].

2. INFOTAB. Appendix I, January 1989 discussion paper, 30 de enero de 1989; http://www.pmdocs.com/getallimg.asp ?DOCID $=2501045258 / 5268 ; \quad$ Bates Number 2501045258-5268.

3. National Center for Tobacco-Free Kids. Hojas doradas, cosecha estéril: los costos de cultivar tabaco. Washington, D.C.: NCTFK; 2001.

4. FAOSTAT-Agriculture. Base de datos de la Organización de las Naciones Unidas para la Agricultura y la Alimentación
(FAO) [base de datos en línea]. [Citado el 4 de octubre de 2002]. Se encuentra en: http://www.fao.org

5. World Health Organization. Tobacco or health: a global status report. Geneva: WHO; 1997.

6. Banco Mundial. La epidemia del tabaquismo: los gobiernos y los aspectos económicos del control del tabaco. Washington, D.C.: BM; 1999.

7. Anónimo. World: Growers reducing tobacco planting, as stocks keep rising. Africa Tobacco 1999;1. [Citado el 19 de abril de 2002] . Se encuentra en URL.

8. Anónimo. Proyecciones a la baja para la cosecha de 2000. La hoja: una publicación informativa de la International Tobacco
Growers 1999;9. [Citado el 19 de abril de 2002]. Se encuentra en URL: http:// www.tobaccoleaf.org/lahoja/art92.htm

9. World Health Organization. The World Health Report 1999. Geneva: WHO; 1999.

10. Pan American Health Organization Health in the Americas. Washington, D.C.: PAHO; 2002.

11. Murray C, Lopez A. Alternative projections of mortality and disability by cause 1990-2020: Global Burden of Disease Study. 1997 May 24;349(9064): 1498-504.

12. Peto R, Lopez AD, Boreham J, Thun M, Heath C. Mortality from smoking in developed countries, 1950-2000. Oxford: Oxford University Press; 1994.

\begin{tabular}{|c|c|}
\hline \multicolumn{2}{|c|}{ World Conference on Tobacco or Health } \\
\hline $\begin{array}{l}\text { Dates: } \\
\text { Location: }\end{array}$ & $\begin{array}{l}\text { 3-8 August } 2003 \\
\text { Helsinki Fair Centre } \\
\text { Helsinki, Fnland }\end{array}$ \\
\hline \multicolumn{2}{|c|}{$\begin{array}{l}\text { The 12th World Conference on Tobacco or Health will deal with the many facets of tobacco } \\
\text { use and tobacco control in different parts of the world. A major theme of the meeting will be the } \\
\text { social process for a smoke-free world and the broad partnership needed for that. Practical } \\
\text { emphasis will also be on the implementation of the World Health Organization's Framework } \\
\text { Convention on Tobacco Control. } \\
\text { The conference will bring together several thousand professionals from around the world } \\
\text { who are involved in tobacco control work. The meeting will take stock of the situation, assess the } \\
\text { state of research, propose action, and map strategies for new initiatives and further success in the } \\
\text { field of tobacco or health. The meeting will have a wide variety of sessions, including plenaries, } \\
\text { main sessions, workshops, professional development sessions, and ancillary and satellite } \\
\text { meetings. The registration fee for the conference is Euro } 590 \text {, or about US\$ } 630 \text {. }\end{array}$} \\
\hline $\begin{array}{r}\text { Infc } \\
\text { Ms. Aira Raudasoja } \\
\text { CongO } \\
\text { POBox 762, 00 } \\
\text { Telephone: } 35894542 \\
\text { E-mail: wctoh20 } \\
\text { Internet: http:// }\end{array}$ & $\begin{array}{l}\text { ormation: } \\
\text { a \& Ms. Hanne Heikkinen } \\
\text { reator OCLtd } \\
\text { 0101 Helsinki, Fnland } \\
190 \text { • Fax: } 358945421930 \\
\text { D03@congcreator.com } \\
\text { www.wctoh2003.org/ }\end{array}$ \\
\hline
\end{tabular}

